\title{
DETERMINATION OF A NONLINEAR SOURCE TERM IN A DIFFUSION EQUATION WITH FINAL OBSERVATIONS
}

\author{
GONGSHENG LI, YICHEN MA, and KAITAI LI
}

Received 25 November 2002

\begin{abstract}
This paper deals with an inverse problem of determining a nonlinear source term in a quasilinear diffusion equation with overposed final observations. Applying integral identity methods, data compatibilities are deduced by which the inverse source problem here is proved to be reasonable and solvable. Furthermore, with the aid of an integral identity that connects the unknown source terms with the known data, a conditional stability is established.
\end{abstract}

2000 Mathematics Subject Classification: 35K30.

1. Introduction. In this paper, we are concerned with an inverse source problem in a diffusion equation

$$
u_{t}-\Delta u=f(u)
$$

where the unknown source term $f$, being a function of the state variable only, is to be determined. Although there are many researches on such inverse source problems from the 1970s (cf., e.g., [1, 3, 4, 7, 8, 10, 12, 13, 14, 15, 16, 17, 18]), it is still worthwhile to consider this kind of problem at least in the following two aspects.

On one hand, the inverse source problem here is based on data measured at the final moment in an experiment that is driven by suitably controlling the boundary value. This leads to a different problem from the studies as in $[1,4,10,15,16,17,18]$ where the source term is often assumed to have an a priori functional form, and the experiment is also different from the problem in $[3,7,8,12,13,14]$ where a state-dependent source term is to be found from data measured on the spatial boundary.

On the other hand, a stability investigation for the nonlinear source term's inversion is presented here. Isakov discussed many stability problems (cf., e.g., [9]), and obtained some wonderful results for linear source term's inversion of parabolic equations. But for nonlinear source terms, there are fewer researches in the literatures we have. In 1982, Lorenzi [12] proved a stability of $W_{\infty}^{\delta}(\delta<1 / 2)$ for nonlinear source $f=f(u)$, and this still seems a better result for inverse problems of nonlinear source term.

Thus, it is also our motivation to deal with (1.1) for determining the nonlinear source term with final observations and pay attention to the stability analysis. Our idea and method are based on integral identities (cf., e.g., [3, 6]), but due to the difference of the problem studied here, integral identities (2.9), (2.15), and (3.1) involving final observations are different from those in paper [3]. These identities expose some compatibility 
information on the direct and inverse problems, which is interesting and meaningful. Moreover, a conditional stability for the nonlinear source term's inversion seems to be an innovation as compared with the known results we have.

The paper is arranged as follows. In Section 2, the direct initial boundary value problem is considered so as to explore necessary compatibilities for the related inverse problem studied in Section 3. Section 3.1 gives an integral identity connecting unknown source terms with known data that are a principal tool in the analysis of the inverse problem. In Section 3.2, using the identity, a minimum of an error functional is proved to be a source term solution showing an existence of the inverse problem (2.1) and (2.3). Finally, in Section 4, a conditional stability for the inverse source problem is established, also with integral identity method. The results imply that data compatibilities can lead to a conditional well-posedness for inverse problems at least for the source determination problem investigated in this paper.

2. The direct initial boundary value problem. For any given $T>0$, denote $D_{T}=$ $\{(x, t): 0<x<1,0<t<T\}$. Consider an initial boundary value problem

$$
\begin{gathered}
u_{t}-u_{x x}=f(u), \quad(x, t) \in D_{T}, \\
u(x, 0)=0, \quad x \in[0,1], \\
u(0, t)=h(t), \quad u(1, t)=0, \quad t \in[0, T],
\end{gathered}
$$

where the functions $f(u)$ and $h$ are assumed to satisfy the following hypotheses:

(A1) $f$ is continuous and piecewise differentiable on $\mathbb{R}$;

(A2) $h \in C[0, \infty), h(0)=0$.

It is well known that the hypotheses (A1) and (A2) are sufficient to imply the local existence of a solution to problem (2.1) [2]. It is also well known that if the local solution satisfies an a priori estimate as follows:

$$
|u(x, t)| \leq M, \quad 0 \leq x \leq 1,0 \leq t \leq T
$$

for any given $T>0$, then it can be extended to a global solution.

Let $u=u(x, t ; f, h)$ denote the solution to problem (2.1) for boundary value $h(t)$ and some source term $f(u)$ satisfying conditions (A1) and (A2). Assume that this solution is known to satisfy an a priori estimate for a fixed $T>0$ so that $u(x, t)$ is a solution in $D_{T}$. Then, $u(x, t)$ will be said to be a solution of the direct initial boundary value problem (2.1).

If the source term $f=f(u)$ is unknown, we have to add some overposed information to solution $u$, which often arrives in an inverse source problem. In this paper, suppose the final observations are known, that is, when $t=T$, we get

$$
u(x, T)=\theta(x), \quad x \in[0,1] .
$$

The function $\theta(x)=: u(x, T ; f, h)$ will be viewed as an output corresponding to the input $h(t)$ in the presence of the source term $f$, and the functions $\theta(x)$ and $h(t)$ are easily seen to satisfy the following conditions of compatibility imposed by the initial 
condition and the equation:

$$
\theta(0)=h(T)>0, \quad \theta(1)=0, \quad h^{\prime}(0)=f(0) .
$$

The following two theorems expose some further conditions of compatibility on the data $\theta$ and $h$, which often lead to a definition of admissible class of data for the inverse problem (2.1) and (2.3).

THEOREM 2.1. Suppose that $u=u(x, t ; f, h)$ satisfies an a priori bound estimate and that the functions $h(t)$ and $f(u)$ satisfy assumptions (A1) and (A2). Suppose further that $h(t)>0$ for $0<t<T$ and that $f(0)=0$. Then it follows that

$$
0=u(x, 0)<u(x, t)<u(x, T)=\theta(x) \quad \text { for }(x, t) \in D_{T} .
$$

Proof. For $u=u(x, t ; f, h)$ and any smooth test function $\varphi(x, t)$, we have

$$
\int_{D_{T}}\left(u_{t}-u_{x x}-f(u)\right) \varphi_{t} d x d t=0
$$

Integration by parts leads to

$$
\begin{aligned}
\int_{D_{T}} & u_{t}\left(\varphi_{t}+\varphi_{x x}+f^{\prime}(u) \varphi\right) d x d t \\
& =\left.\int_{0}^{T}\left(u_{x} \varphi_{t}-u \varphi_{x t}\right)\right|_{x=0} ^{x=1} d t+\left.\int_{0}^{1}\left(u \varphi_{x x}+f(u) \varphi\right)\right|_{t=0} ^{t=T} d x .
\end{aligned}
$$

Now suppose $\varphi=\varphi(x, t)$ solves the adjoint problem

$$
\begin{gathered}
\varphi_{t}+\varphi_{x x}+f^{\prime}(u) \varphi=G(x, t) \quad \text { in } D_{T}, \\
\varphi(0, t)=\varphi(1, t)=0, \quad 0 \leq t \leq T, \\
\varphi(x, T)=0, \quad 0 \leq x \leq 1 .
\end{gathered}
$$

Note that problem (2.8) imposes a condition on $\varphi(x, t)$ at $t=T$, it is backward in time but is well-posed due to the reverse parabolic character of the partial differential equation.

If $\varphi(x, t)$ solves (2.8), together with the initial boundary value conditions and hypotheses (A1) and (A2), equality (2.7) reduces to

$$
\int_{D_{T}} u_{t} G(x, t) d x d t=\int_{0}^{T} h(t) \varphi_{x t}(0, t) d t
$$

For $f$ satisfying hypothesis (A1) and $u$ a priori bounded, it follows that $f^{\prime}(u(x, t))$ is bounded for $(x, t) \in D_{T}$. Then the maximum-minimum principle can be applied to the adjoint problem (2.8) to conclude that if $G(x, t)$ is nonnegative but is otherwise arbitrary, then $\varphi(x, t)$ is negative in $D_{T}$. However, if $\varphi(x, t)<0$ in $D_{T}$, then $\varphi_{x}(0, t)<0$ for $0<t<T$, and noting that $\varphi_{x}(0, T)=0$, we can deduce $\varphi_{x t}(0, t)>0$.

On the other hand, by the hypothesis $h(t)>0$ for $0<t<T$, we know that the righthand side of (2.9) is positive. Since $G=G(x, t)$ is nonnegative but otherwise arbitrary, it follows that if there was any positive measure subset of $D_{T}$ where $u_{t}(x, t)$ was zero 
or negative, then a contradiction of (2.9) could be achieved by choosing the support $G$ in this positive measure set. This proves that for each $0<x<1$, there is $u_{t}(x, t)>0$ for $0<t<T$, that is,

$$
0=u(x, 0)<u(x, t)<u(x, T)=\theta(x) \text { for }(x, t) \in D_{T} .
$$

THEOREM 2.2. Under the conditions of Theorem 2.1, if, in addition, $f \geq 0, f \not \equiv$ for $0 \leq u \leq M$ for some $M>0$, then it follows that $h^{\prime}(t)>0$ for $0<t<T$, and $\theta^{\prime}(x)<0$ for $0<x<1$.

Proof. By Theorem 2.1, we can denote $M=\max _{x \in[0,1]} \theta(x)$ as an a priori bound for solution $u$. So, the assumption $0 \leq u \leq M$ is reasonable. First, we will prove $h^{\prime}(t)>0$ for $0<t<T$.

By equality,

$$
\int_{D_{T}}\left(u_{t}-u_{x x}-f(u)\right) \varphi_{x} d x d t=0
$$

and with a similar method as in Theorem 2.1, we have

$$
\begin{aligned}
\int_{D_{T}} u_{x}\left(\varphi_{t}+\varphi_{x x}+f^{\prime}(u) \varphi\right) d x d t= & -\int_{0}^{T}\left[h(t) \varphi_{t}(0, t)+f(h(t)) \varphi(0, t)\right] d t \\
& -\int_{0}^{1} \theta(x) v(x) d x .
\end{aligned}
$$

Here, the test function $\varphi(x, t)$ is set to solve the following adjoint problem instead of (2.8):

$$
\begin{gathered}
\varphi_{t}+\varphi_{x x}+f^{\prime}(u) \varphi=0 \quad \text { in } D_{T}, \\
\varphi_{x}(0, t)=\varphi_{x}(1, t)=0, \quad 0 \leq t \leq T, \\
\varphi(x, T)=v(x), \quad 0 \leq x \leq 1,
\end{gathered}
$$

where the input data $v(x)$ satisfies $v(0)=0$ but is otherwise arbitrary.

Note that when $h(0)=0, \varphi(0, T)=v(0)=0$, we can get

$$
\int_{0}^{T} h(t) \varphi_{t}(0, t) d t=-\int_{0}^{T} h^{\prime}(t) \varphi(0, t) d t
$$

hence, the integral identity reduces further to

$$
\int_{0}^{1} \theta(x) v(x) d x=\int_{0}^{T}\left[h^{\prime}(t)-f(h(t))\right] \varphi(0, t) d t .
$$

Now, the initial data $v(x)$ in (2.13) is additionally required to be monotoneincreasing and positive for $0 \leq x \leq 1$. By virtue of this additional condition, the solution $\varphi$ of adjoint problem (2.13) is to be positive. In fact, letting $\tau=T-t$, problem 
(2.13) can be viewed as an ordinary Neumann boundary value problem as shown below:

$$
\begin{gathered}
\varphi_{t}-\varphi_{x x}-f^{\prime}(u) \varphi=0 \quad \text { in } D_{T}, \\
\varphi_{x}(0, t)=\varphi_{x}(1, t)=0, \quad 0 \leq t \leq T, \\
\varphi(x, 0)=v(x), \quad 0 \leq x \leq 1 .
\end{gathered}
$$

Hence, a version of the extended maximum-minimum principle [2] shows that the minimum of solution $\varphi(x, t)$ of problem (2.16) must occur at $t=0$, which implies $\varphi(x, t) \geq v(0)=0$ for $0<x<1$ and $0<t<T$. Combining this with $\theta(x)>0$ for $0<x<1$ and the hypothesis (A1), it follows from (2.15) that $h^{\prime}(t)>f(h(t)) \geq 0$ in $(0, T)$ for $T$ at least as large as the number $h^{-1}(M)$.

We follow the hypothesis on the data $v=v(x)$ as in the above discussions, and furthermore, let

$$
\int_{0}^{x} v(s) d s=w(x), \quad 0 \leq x \leq 1
$$

That is, $v(x)=w^{\prime}(x), 0 \leq x \leq 1$, and $w(x)$ satisfies $w(x) \geq 0, w(0)=0$. Thus, replacing $v(x)$ with $w^{\prime}(x)$ in (2.15), we have

$$
\int_{0}^{1} \theta(x) w^{\prime}(x) d x=\int_{0}^{T}\left[h^{\prime}(t)-f(h(t))\right] \varphi(0, t) d t>0 .
$$

Noting that

$$
\int_{0}^{1} \theta(x) w^{\prime}(x) d x=\left.\theta(x) w(x)\right|_{x=0} ^{x=1}-\int_{0}^{1} w(x) \theta^{\prime}(x) d x=-\int_{0}^{1} w(x) \theta^{\prime}(x) d x,
$$

we get

$$
\int_{0}^{1} w(x) \theta^{\prime}(x) d x<0
$$

which implies $\theta^{\prime}(x)<0$, that is, $0=\theta(1)<\theta(x)<\theta(0)$ for $0<x<1$.

3. The inverse source problem. By Theorems 2.1 and 2.2, inverse source problem (2.1) and (2.3) should be investigated under the following compatibility conditions on data functions $h(t)$ and $\theta(x)$ :

$\left(\mathrm{A} 2^{\prime}\right) h \in C[0, T], h(0)=0$, and $h(t) \geq 0, h^{\prime}(t)>0$ for $0<t<T$;

(A3) $\theta \in C[0,1], \theta(1)=0$, and $\theta(x) \geq 0, \theta^{\prime}(x)<0$ for $0<x<1$.

Consequently, hypothesis (A1) should be replaced by

$\left(\mathrm{A}^{\prime}\right) f$ is continuous and piecewise differentiable on $\mathbb{R}^{+}, f(0)=0, f(u) \geq 0, f(u) \not \equiv 0$, for $u \in[0, M]$ where $M=h(T)=\theta(0)$ for some $T>0$.

These conditions are consistent with $h(t)$ and $\theta(x)$, being respectively, the values of $u(0, t)$ and $u(x, T)$ associated with a solution of (2.1) for $f=f(u)$ satisfying assumption (A1'), that is, the conditions on $h$ and $\theta$ are necessary if $h, \theta$, and $f$ are related by $\theta(x)=u(x, T ; f, h)$. The inverse problem is defined as the problem of determining $f(u)$ on an interval $[0, h(T)]$ from the data $h(t)$ and $\theta(x)$. The source term $f(u)$ is said 
to be a solution of this inverse problem if $h, \theta$, and $f$ are related by $\theta=u(x, T ; f, h)$, $0 \leq x \leq 1$.

Now we are to construct a corresponding relation connecting the unknown source terms with the additional data.

3.1. Construction of an integral identity. The following integral identity reflects a corresponding relation of variations of the unknown source functions with changes of the additional observations.

THEOREM 3.1. Assume conditions (A1) and (A2) are satisfied and let $u_{i}=u\left(x, t ; f_{i}, h\right)$, $\theta_{i}=u_{i}(x, T)(i=1,2)$, then it follows that

$$
\int_{0}^{1}\left(\theta_{1}-\theta_{2}\right) v(x) d x=\int_{D_{T}}\left[f_{1}\left(u_{2}(x, t)\right)-f_{2}\left(u_{2}(x, t)\right)\right] \varphi(x, t) d x d t,
$$

where $\varphi(x, t)$ denotes a solution of a suitable adjoint problem with input data $v(x)$.

Proof. Denote $U=u_{1}-u_{2}$, and note that $u_{1}=u\left(x, t ; f_{1}, h\right)$ and $u_{2}=u\left(x, t ; f_{2}, h\right)$, both satisfying the initial boundary value problem (2.1), then we have

$$
\begin{gathered}
U_{t}-U_{x x}=f_{1}\left(u_{1}\right)-f_{2}\left(u_{2}\right), \quad(x, t) \in D_{T}, \\
U(x, 0)=0, \quad x \in[0,1], \\
U(0, t)=0, \quad U(1, t)=0, \quad t \in[0, T], \\
U(x, T)=\theta_{1}-\theta_{2}, \quad x \in[0,1] .
\end{gathered}
$$

For the test function $\varphi(x, t)$, with a similar method as in Section 2, we have

$$
\int_{D_{T}}\left(U_{t}-U_{x x}\right) \varphi d x d t=\int_{D_{T}}\left[f_{1}\left(u_{1}\right)-f_{2}\left(u_{2}\right)\right] \varphi d x d t
$$

Integration by part leads to

$$
\begin{gathered}
-\int_{D_{T}} U\left[\varphi_{t}+\varphi_{x x}\right] d x d t+\int_{0}^{1} U(x, T) \varphi(x, T) d x \\
-\int_{0}^{T}\left[U_{x}(1, t) \varphi(1, t)-U_{x}(0, t) \varphi(0, t)\right] d t \\
=\int_{D_{T}}\left[p(x, t) U(x, t)+\Delta f\left(u_{2}\right)\right] \varphi(x, t) d x d t
\end{gathered}
$$

where

$$
\begin{gathered}
p(x, t)=f_{1}^{\prime}\left(u_{2}+s\left(u_{1}-u_{2}\right)\right), \quad 0<s<1, \\
\Delta f(u)=f_{1}(u)-f_{2}(u) .
\end{gathered}
$$

If $\varphi=\varphi(x, t)$ is chosen to be the solution of the adjoint problem

$$
\begin{gathered}
\varphi_{t}+\varphi_{x x}+p(x, t) \varphi(x, t)=0, \quad(x, t) \in D_{T}, \\
\varphi(0, t)=\varphi(1, t)=0, \quad t \in[0, T], \\
\varphi(x, T)=v(x), \quad x \in[0,1],
\end{gathered}
$$


then noting that $U(x, T)=\theta_{1}(x)-\theta_{2}(x)$, together with the above computations, the proof is completed.

REMARK 3.2. The problem (3.6) is backward in time, but by the transformation $T=$ $T-t$, and also denoting $\tau$ as $t$, the problem can be reduced to an ordinary initial boundary value problem of parabolic equation as follows:

$$
\begin{gathered}
\varphi_{t}-\varphi_{x x}-p(x, t) \varphi(x, t)=0, \quad(x, t) \in D_{T}, \\
\varphi(0, t)=\varphi(1, t)=0, \quad t \in[0, T], \\
\varphi(x, 0)=v(x), \quad x \in[0,1] .
\end{gathered}
$$

In the following sections, we will consider problem (3.7) instead of (3.6) when we deal with integral identities.

3.2. Existence. By the above discussions, we should find a solution of inverse problem (2.1) and (2.3) under the prescribing condition (A1'). That is to say, an admissible class of source terms can be defined with assumption $\left(\mathrm{A} 1^{\prime}\right)$. For convenience, denote this admissible set by

$$
S_{\mathrm{ad}}=\left\{f: f=f(u) \text { satisfying assumption }\left(\mathrm{A} 1^{\prime}\right)\right\} .
$$

So, for any $\bar{f} \in S_{\text {ad }}$, the direct problem (2.1) has a unique solution denoted by $\bar{u}=$ $u(x, t, \bar{f}, h)$. Then a corresponding relation from the input $\{f\}$ to the output $\{\theta\}$ is well defined as follows:

$$
\left.(K f)\right|_{t=T}=\theta(x)
$$

On actual occasions however, for any given $\bar{f}$, we are generally to seek for a $\bar{u}$ such that $\left.\bar{u}\right|_{t=T}$ approaches to the overposed data $\theta$ as close as possible. That is to say, inverse problems can often be reformulated as optimization problems in which an error functional based on the output data is to be minimized over the admissible inputs. For example, define an error functional by

$$
J[f]=\int_{0}^{1}(u(x, T ; f, h)-\theta(x))^{2} d x .
$$

It will be shown that a minimum of the functional $J$ over the admissible class $S_{\text {ad }}$ is also a source term solution of the inverse problem (2.1) and (2.3). At first, thanks to the convexity of $J$, and by minimal existence theorems of nonlinear functional (cf. [5], for instance), the following assertion is obviously valid.

LEMMA 3.3. There is at least one minimum for error functional $J$ over $S_{\text {ad }}$.

Now we will show that the minimum is indeed a corresponding source term solution to inverse problem (2.1) and (2.3). For this reason, the gradient of functional $J$ which can be worked out using identity (3.1) is needed. 
LEMмA 3.4. For functional $J$ defined by expression (3.10), the gradient is

$$
\nabla J[f]=\varphi(x, t),
$$

where $\varphi(x, t)$ is the solution of adjoint problem (3.6) with $v(x)=2(u(x, T ; f, h)-\theta(x))$.

Proof. By expression (3.10), the first variation of functional $J$ is

$$
\delta J[f, \delta f]=\int_{0}^{1} 2(u(x, T ; f, h)-\theta(x)) \delta u(x, T) d x,
$$

where

$$
\delta u(x, T)=u(x, T ; f+\delta f, h)-u(x, T ; f, h) .
$$

Applying integral identity (3.1), and setting $f_{1}=f+\delta f, f_{2}=f$, there exists

$$
\delta J[f, \delta f]=\int_{0}^{1} \delta u(x, T) v(x) d x=\int_{D_{T}} \varphi(x, t) \delta f d x d t=(\varphi(x, t), \delta f) .
$$

Noting that

$$
\delta J[f, \delta f]=(\nabla J[f], \delta f),
$$

the conclusion is valid.

THEOREM 3.5. Assume conditions (A2') and (A3) are satisfied, then the minimal point of the functional $J$ over $S_{\mathrm{ad}}$ is just a source term solution of inverse problem (2.1) and (2.3).

Proof. Obviously, it is only to testify that expression (2.3) is valid. If $f_{0}$ is the minimal point of $J$, then we have

$$
\varphi(x, t)=\nabla J\left[f_{0}\right] \equiv 0 \quad \forall(x, t) \in D_{T} .
$$

Noting that $\varphi(x, t)$ is the solution of problem (3.6) with $v(x)=2\left(u\left(x, T ; f_{0}, h\right)-\theta(x)\right)$, and according to the theory of general parabolic equations, when the solution of problem (3.6) is identically equal to zero, the unique data $v(x)$ must also be zero. Hence, we have

$$
u\left(x, T ; f_{0}, h\right)=\theta(x), \quad x \in[0,1],
$$

which completes the proof.

4. Conditional stability. Suppose $\left(u_{i}, f_{i}\right), i=1,2$, are two pairs of solutions of the inverse problem (2.1) and (2.3) corresponding to data $h_{i}, \theta_{i}(i=1,2)$. Completely similar to the construction of integral identity (3.1), we have

$$
\begin{array}{r}
\int_{0}^{1}\left(\theta_{1}-\theta_{2}\right) v(x) d x+\int_{0}^{T}\left(h_{2}-h_{1}\right) \varphi_{x}(0, t) d t \\
=\int_{D_{T}}\left(f_{1}\left(u_{2}\right)-f_{2}\left(u_{2}\right)\right) \varphi(x, t) d x d t
\end{array}
$$


where $\varphi=\varphi(x, t)$ is still the solution of the auxiliary problem (3.6) determined by the input data $v=v(x)$.

As we know, a stability should be established with a suitable norm. By equality (4.1), we can see that if the known data have some perturbations, the source term must have corresponding changes.

Considering the adjoint problem (3.7), we can see that by suitably controlling the inputs data $v$, a desired solutions set $\{\varphi(x, t ; v)\}$ can be obtained with which a conditional stability will be established. The following lemma is necessary.

LEMmA 4.1. Suppose $p(x, t) \in L^{\infty}$, then the solutions set $\{\varphi=\varphi(x, t ; v)\}$ of the adjoint problem (3.7) is dense in $L^{2}\left(D_{T}\right)$ as the initial input $v=v(x)$ ranges over $L^{2}(0,1)$.

Proof [11]. For convenience denote the operator $A=-\partial^{2} / \partial x^{2}-p I$. Then by (3.7), we have

$$
\varphi_{t}+A \varphi=0 .
$$

Suppose $\psi(x, t) \in L^{2}\left(D_{T}\right)$ is such that

$$
(\varphi(x, t ; v), \psi)_{L^{2}}=0 \quad \forall v \in L^{2}(0,1) .
$$

We introduce $\xi=\xi(x, t)$ as the solution of

$$
\begin{gathered}
-\xi_{t}+A^{*} \xi=\psi, \quad(x, t) \in D_{T}, \\
\xi(0, t)=\xi(1, t)=0, \quad t \in[0, T], \\
\xi(x, T)=0, \quad x \in[0,1],
\end{gathered}
$$

where $A^{*}$ denotes the dual operator of $A$. Then using conditions of (3.7) and (4.4), and integration by parts, leads to

$$
\begin{aligned}
(\varphi, \psi)_{L^{2}} & =\int_{D_{T}} \varphi \psi d x d t=\int_{D_{T}}\left(-\xi_{t}+A^{*} \xi\right) \varphi d x d t \\
& =\int_{0}^{1} \xi(x, 0) v(x) d x+\int_{D_{T}}\left(\varphi_{t}+A \varphi\right) \xi d x d t=\int_{0}^{1} \xi(x, 0) v(x) d x,
\end{aligned}
$$

together with (4.3), it follows that

$$
\int_{0}^{1} \xi(x, 0) v(x) d x=0 \quad \forall v \in L^{2}(0,1),
$$

hence $\xi(x, 0)=0$. But noting that problem (4.4) is backward in time, we know that $\xi \equiv 0$ from the backward uniqueness property, and hence $\psi=0$. This implies that the space generated by $\varphi(x, t ; v)$ as $v$ ranges over $L^{2}(0,1)$ is dense in $L^{2}\left(D_{T}\right)$. The proof is completed.

Using this lemma, we can prove Lemma 4.2.

LEMMA 4.2. For the problem (3.7), if $p(x, t) \in L^{\infty}$, then there exist an admissible inputs set $\mathscr{V} \subset L^{2}(0,1)$ and the corresponding solutions set

$$
\Phi=\{\varphi(x, t ; v), v \in \mathscr{V}\} \subset L^{2}\left(D_{T}\right),
$$


and a positive constant $c_{1}$ only depending on the domain $D_{T}$ and the inputs $v$ such that

$$
\|\varphi\|_{2} \geq c_{1}\|v\|_{2}, \quad v \in \mathscr{V}
$$

where $\mathscr{V}$ is given in the proof of this lemma.

Proof. By the fact that the solutions set $\{\varphi=\varphi(x, t ; v)\}$ of problem (3.7) is dense in $L^{2}\left(D_{T}\right)$ as the boundary control $v(x)$ ranges over $L^{2}(0,1)$, we know that for any $\delta>0$, there exists $v \in L^{2}(0,1)$ such that

$$
\|\varphi(x, t ; v)\|_{2} \geq \delta
$$

Contrarily, if there exists $\delta_{1}>0$ such that

$$
\|\varphi(x, t ; v)\|_{2}<\delta_{1}
$$

for any $v \in L^{2}(0,1)$, then set $\phi \in L^{2}\left(D_{T}\right)$ satisfying the condition $\|\phi\|_{2} \geq 2 \delta_{1}$. By Lemma 4.1 , we know that for the above $\phi$, there is at least one $\bar{v} \in L^{2}(0,1)$ and the corresponding solution $\varphi(x, t ; \bar{v})$ to problem (3.7) such that

$$
\|\varphi(x, t, \bar{v})-\phi\|_{2} \leq \delta_{1}
$$

Then, we can get

$$
\|\varphi(x, t ; \bar{v})\|_{2} \geq\|\phi\|_{2}-\delta_{1} \geq \delta_{1}>0
$$

which is a contradiction with (4.10), showing that (4.9) is valid.

Now, by the above discussions, we set a positive constants series $\left\{\delta_{n}, n \in \mathbb{N}\right\}$, and a corresponding series of inputs $\left\{v_{n}, n \in \mathbb{N}\right\} \subset L^{2}(0,1)$ such that

$$
\left\|\varphi\left(x, t ; v_{n}\right)\right\|_{2} \geq \delta_{n}
$$

Denote

$$
\begin{array}{cc}
\mathscr{V}=\left\{v_{n}, n \in \mathbb{N}\right\}, & \Phi=\{\varphi(x, t ; v), v \in \mathscr{V}\}, \\
\bar{\delta}=\inf _{n \in \mathbb{N}}\left\{\delta_{n}\right\}, & M_{v}=\sup _{n \in \mathbb{N}}\left\{\left\|v_{n}\right\|_{2}\right\} .
\end{array}
$$

Then set $c_{1}=\bar{\delta} / M_{v}$. We have

$$
c_{1}\|v\|_{2} \leq \bar{\delta} / M_{v} \cdot M_{v}=\bar{\delta} \leq\|\varphi(x, t ; v)\|_{2}
$$

for $v \in \mathscr{V}$, which completes the proof of the lemma.

REMARK 4.3. This lemma gives a continuously dependent relation of $\varphi$ on function $p=p(x, t)$ and input data $v=v(x)$. Under the assumption (A1'), it follows that 
$f^{\prime}(u(x, t))$ is bounded, which implies that $p(x, t)$ is bounded for $(x, t) \in D_{T}$, that is, Lemma 4.2 is valid.

Now, noting the identity (4.1), we define a bilinear form

$$
B(f, \varphi): L^{2}\left(D_{T}\right) \times L^{2}\left(D_{T}\right) \rightarrow \mathbb{R}
$$

by

$$
B(f, \varphi)=\int_{D_{T}} f(u(x, t)) \varphi(x, t) d x d t
$$

Obviously, $B(f, \varphi)$ is bounded. For the above solutions set $\Phi$, by supplementing zero element, we introduce a subspace $W=\Phi \cup\{0\} \subset L^{2}\left(D_{T}\right)$, and define a norm as follows:

$$
\|f\|_{2, \varphi}=\sup _{\varphi \in W, \varphi \neq 0}\left\{|B(f, \varphi)| /\|\varphi\|_{2}\right\}
$$

By the above norm (4.18), a conditional stability, which can be named confined $L^{2}$ stability for the inverse source term problem, can be constructed as follows.

THEOREM 4.4. Under the assumptions of Theorem 3.5, suppose $\left(u_{i}, f_{i}\right), i=1,2$ are two pairs of solutions of the inverse problem (2.1) and (2.3) corresponding to data $h_{i}, \theta_{i}$ $(i=1,2)$. Then for $v \in \mathcal{V}$, there exists a positive constant $c$ independent of data $\theta$ and $h$ such that

$$
\left\|f_{1}-f_{2}\right\|_{2, \varphi} \leq c\left(\left\|\theta_{1}-\theta_{2}\right\|_{2}+\left\|h_{1}-h_{2}\right\|_{2}\right) .
$$

Proof. On the base of the above discussions, the proof is trivial. In fact, by identity (4.1), and applying Schwartz inequality, we have

$$
\begin{aligned}
\left\|f_{1}-f_{2}\right\|_{2, \varphi} \leq & \left\|\theta_{1}-\theta_{2}\right\|_{2} \sup _{\varphi \in W, \varphi \neq 0}\left\{\|v\|_{2} /\|\varphi\|_{2}\right\} \\
& +\left\|h_{1}-h_{2}\right\|_{2} \sup _{\varphi \in W, \varphi \neq 0}\left\{\left\|\varphi_{x}(0, t)\right\|_{2} /\|\varphi\|_{2}\right\} \\
= & I_{1}+I_{2} .
\end{aligned}
$$

First, paying attention to Lemma 4.2, we have

$$
I_{1} \leq 1 / c_{1} \cdot\left\|\theta_{1}-\theta_{2}\right\|_{2}
$$

Second, by general parabolic theory, we know that if $p(x, t)$ is bounded, then the adjoint problem (3.7) has a unique solution $\varphi=\varphi(x, t ; v)$ for each $v \in L^{2}(0,1)$, and there exists a constant $c_{2}>0$ such that

$$
\left\|\varphi_{x}\right\|_{2} \leq c_{2}\|v\|_{2} .
$$


Therefore, we can get

$$
I_{2} \leq c_{2}\left(\|v\|_{2} /\|\varphi\|_{2}\right) \cdot\left\|h_{1}-h_{2}\right\|_{2} \leq c_{2} / c_{1} \cdot\left\|h_{1}-h_{2}\right\|_{2}
$$

Set $c=\max \left\{1 / c_{1}, c_{2} / c_{1}\right\}$. Combining $I_{1}$ with $I_{2}$, it follows that the proof is over.

REMARK 4.5. This theorem shows a conditional $L^{2}$ stability for the inverse source problem (2.1) and (2.3). As a corollary, by this theorem, we know that a uniqueness for the inverse source problem can be easily deduced by the stability estimate (4.19).

ACKNOWLEDGMENTs. Many thanks to the referees and editors for their valuable suggestions. The authors also give their best wishes to Prof. Tan and Prof. Cheng of Fudan University. The first author was supported by the visiting funds from the Ministry of Education of China, he thanks the Institute of Mathematics at Fudan University for the hospitality during his visit. This project was supported by the Natural Science Foundation (NSF) of Shandong Province Grant Y2001E03.

\section{REFERENCES}

[1] I. Bushuyev, Global uniqueness for inverse parabolic problems with final observation, Inverse Problems 11 (1995), no. 4, L11-L16.

[2] J. R. Cannon, The One-Dimensional Heat Equation, Encyclopedia of Mathematics and Its Applications, vol. 23, Addison-Wesley Publishing, Massachusetts, 1984.

[3] J. R. Cannon and P. DuChateau, Structural identification of an unknown source term in a heat equation, Inverse Problems 14 (1998), no. 3, 535-551.

[4] M. Choulli and M. Yamamoto, An inverse parabolic problem with non-zero initial condition, Inverse Problems 13 (1997), no. 1, 19-27.

[5] K. Deimling, Nonlinear Functional Analysis, Springer-Verlag, Berlin, 1985.

[6] P. DuChateau, Monotonicity and invertibility of coefficient-to-data mappings for parabolic inverse problems, SIAM J. Math. Anal. 26 (1995), no. 6, 1473-1487.

[7] P. DuChateau and W. Rundell, Unicity in an inverse problem for an unknown reaction term in a reaction-diffusion equation, J. Differential Equations 59 (1985), no. 2, 155-164.

[8] S. Gatti, An existence result for an inverse problem for a quasilinear parabolic equation, Inverse Problems 14 (1998), no. 1, 53-65.

[9] V. Isakov, Inverse Problems for Partial Differential Equations, Applied Mathematical Sciences, vol. 127, Springer-Verlag, New York, 1998.

[10] Some inverse problems for the diffusion equation, Inverse Problems 15 (1999), no. 1, 3-10.

[11] J.-L. Lions, Optimal Control of Systems Governed by Partial Differential Equations, Die Grundlehren der mathematischen Wissenschaften, vol. 170, Springer-Verlag, New York, 1971.

[12] A. Lorenzi, An inverse problem for a semilinear parabolic equation, Ann. Mat. Pura Appl. (4) 131 (1982), 145-166.

[13] A. Nanda and P. C. Das, Determination of the source term in the heat conduction equation, Inverse Problems 12 (1996), no. 3, 325-339.

[14] M. Pilant and W. Rundell, Fixed point methods for a nonlinear parabolic inverse coefficient problem, Comm. Partial Differential Equations 13 (1988), no. 4, 469-493.

[15] R. Riganti and E. Savateev, Solution of an inverse problem for the nonlinear heat equation, Comm. Partial Differential Equations 19 (1994), no. 9-10, 1611-1628.

[16] W. Rundell, The determination of a parabolic equation from initial and final data, Proc. Amer. Math. Soc. 99 (1987), no. 4, 637-642. 
[17] E. G. Savateev and R. Riganti, Inverse problem for the nonlinear heat equation with the final overdetermination, Math. Comput. Modelling 22 (1995), no. 1, 29-43.

[18] M. Tadi, Inverse heat conduction based on boundary measurement, Inverse Problems 13 (1997), no. 6, 1585-1605.

Gongsheng Li: School of Mathematics and Information Science, Shandong University of Technology, Zibo, Shandong 255049, China

E-mail address: 1igs@sdut.edu.cn

Yichen Ma: School of Sciences, Xi'an Jiaotong University, Xi'an, Shannxi 710049, China

E-mail address: ycma@mai1.xjtu.edu.cn

Kaitai Li: School of Sciences, Xi'an Jiaotong University, Xi'an, Shannxi 710049, China

E-mail address: kt1i@mai1.xjtu.edu.cn 


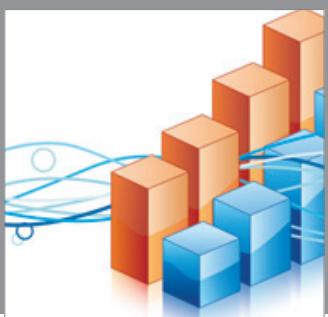

Advances in

Operations Research

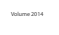

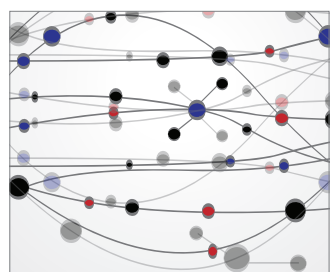

\section{The Scientific} World Journal
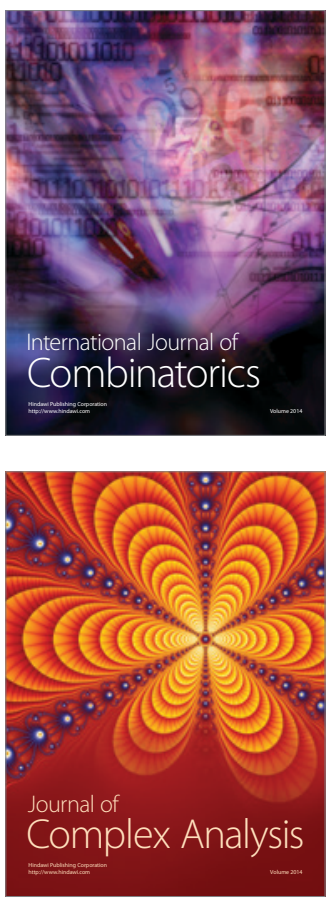

International Journal of

Mathematics and

Mathematical

Sciences
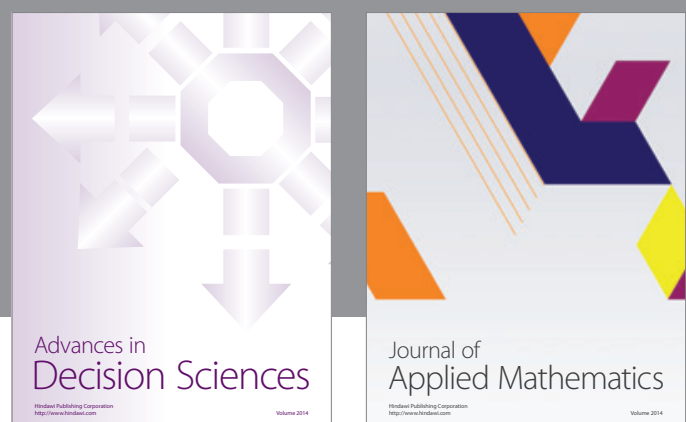

Journal of

Applied Mathematics
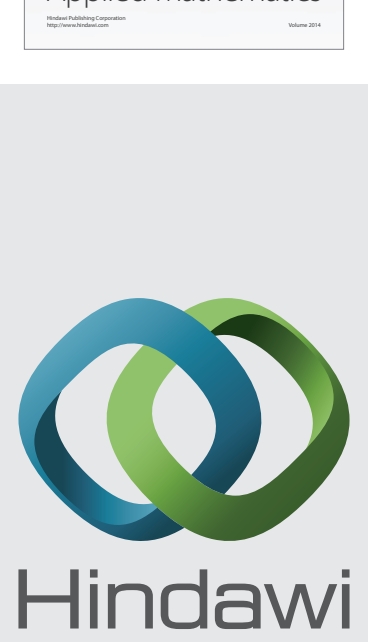

Submit your manuscripts at http://www.hindawi.com
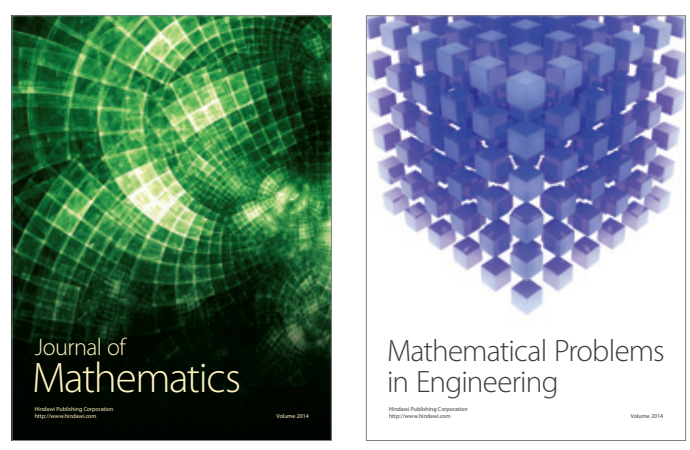

Mathematical Problems in Engineering
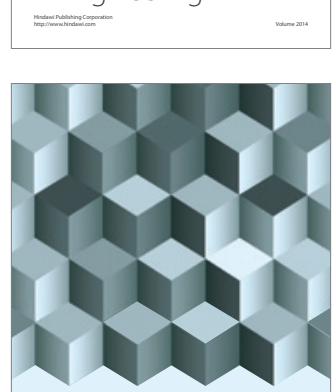

Journal of

Function Spaces
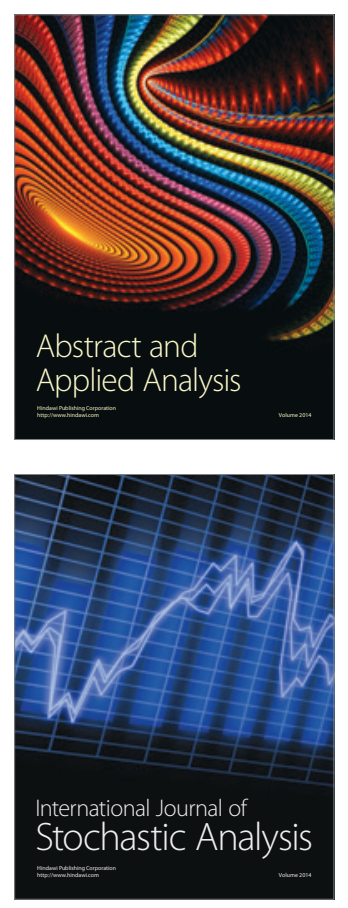

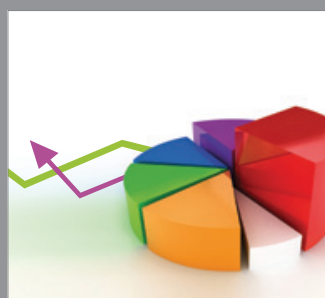

ournal of

Probability and Statistics

Promensencen
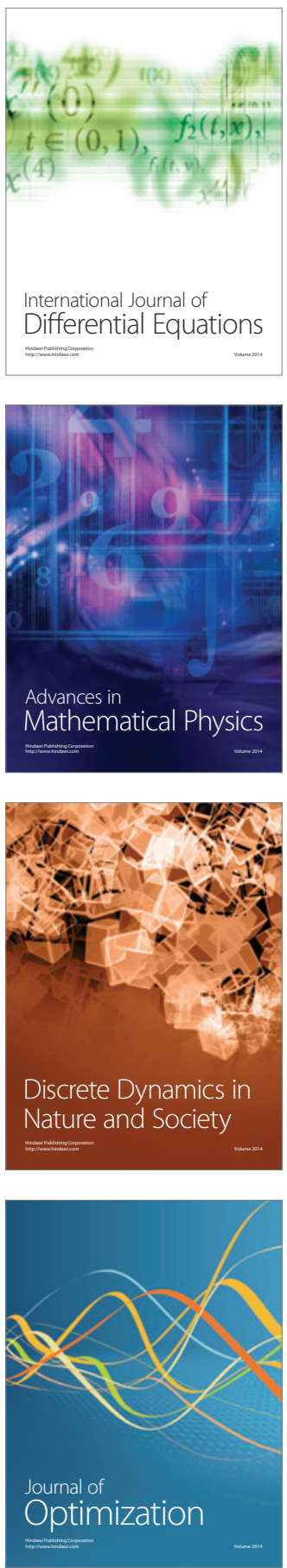